\title{
A PROBABILISTIC APPROACH TO A SYSTEM OF INTEGRAL EQUATIONS ${ }^{1}$
}

\author{
LEO A. GOODMAN
}

The following system of integral equations is of some statistical interest:

$$
\begin{array}{r}
G_{n}(y)=\int_{0}^{y} \frac{e^{-\omega} \omega^{n \alpha-1}}{\Gamma(n \alpha)} d \omega=\int_{0}^{o(y)} H_{n-1}(g(y)-z ; g) d H_{1}(z ; g) \\
\text { for } n=1,2, \cdots,
\end{array}
$$

where $g(x) \geqq 0$ is an increasing continuous function of $x \geqq 0, H_{0}(x ; g)$ $=1$, and

$$
H_{n}(x ; g)=\int_{0}^{x} H_{n-1}(x-z ; g) d H_{1}(z ; g) \text {. }
$$

We shall show by a probabilistic approach (distribution functions, the moment problem, etc.) that the only functions satisfying this system of equations for fixed $\alpha, 0<\alpha \leqq 2$, are $g(x)=c x$, where $c$ is a constant. It will then be seen that this result is a probabilistic analogue of the well known Cauchy functional equation. Also an application of this result to statistics is presented.

THEOREM 1. If $g(x)$ satisfies the Cauchy functional equation

$$
g(x+y)=g(x)+g(y),
$$

then $g(x)$ satisfies (1).

Proof. For fixed $n$, we may define a random variable $Y$ by the relation $\operatorname{Pr}\{Y \leqq y\}=G_{n}(y)$. Since $H_{1}(v ; g)=\int_{0}^{f(v)}\left(e^{-\omega} \omega^{\alpha-1} / \Gamma(\alpha)\right) d \omega$, where $f(v)$ is the inverse function of $g(x)$, we may define a random variable $V$ by the relation

$$
\operatorname{Pr}\{V \leqq v\}=H_{1}(v ; g) .
$$

Hence $H_{n}(v ; g)$ may be regarded as the distribution of the sum of $n$ independent random variables $V_{i}$ each distributed as $V$. Equation (1) then states

$$
\begin{aligned}
\operatorname{Pr}\{Y \leqq y\} & =\operatorname{Pr}\left\{V_{1}+V_{2}+\cdots+V_{n} \leqq g(y)\right\} \\
& =\operatorname{Pr}\left\{f\left(V_{1}+V_{2}+\cdots+V_{n}\right) \leqq y\right\} .
\end{aligned}
$$

Received by the editors April 26, 1951.

1 This research was prepared under an Office of Naval Research contract. 
When $n=1$, we may write $Y=f\left(V_{1}\right)$. By the addition theorem for gamma distributions, we have

$$
\begin{aligned}
\operatorname{Pr}\left\{f\left(V_{1}\right)+f\left(V_{2}\right)+\cdots\right. & \left.+f\left(V_{n}\right) \leqq y\right\}=G_{n}(y) \\
& =\operatorname{Pr}\left\{f\left(V_{1}+V_{2}+\cdots+V_{n}\right) \leqq y\right\} .
\end{aligned}
$$

Since when $g$ satisfies (2), so does $f$, a fortiori (4) and (1) are satisfied by $f$ and $g$. Q.E.D.

Theorem 2. The only functions satisfying (1) for fixed $\alpha, 0<\alpha \leqq 2$, are $g(x)=c x$, where $c$ is a constant.

Proof. We use the notation developed in the proof of Theorem 1. Then $g(Y)$ is a random variable and by (3) has the same distribution function as the sum of $n$ independent random variables $V_{i}$. Let $\phi(t)$ $=\int_{0}^{\infty} e^{-t x} d G_{1}(f(x))$, for fixed $t>0$. Then by the fundamental property of moment generating functions, $\int_{0}^{\infty} e^{-t x} d G_{n}(f(x))=\phi^{n}(t)$. Hence, we have the system of equations

$$
\int_{0}^{\infty} e^{-t g(\omega)-\omega} \frac{\omega^{n \alpha-1}}{\Gamma(n \alpha)} d \omega=\phi^{n}(t) .
$$

Let us define a new random variable $Z$ which has the distribution function

$$
\operatorname{Pr}\{Z \leqq z\}=\int_{0}^{z} \frac{e^{-t_{g}(\omega)-\omega}}{\phi \Gamma(\alpha)} \omega^{\alpha-1} d \omega, \quad \text { where } \phi=\phi(t) .
$$

Hence by (5) we have that the $n-1$ moment of $Z^{\alpha} / \phi$ is equal to $\Gamma(n \alpha) / \Gamma(\alpha)$. It can easily be seen that $1, \Gamma(2 \alpha) / \Gamma(\alpha), \Gamma(3 \alpha) / \Gamma(\alpha), \cdots$ is the sequence of moments of the density function

$$
\frac{e^{-h^{1 / \alpha}}}{\Gamma(\alpha+1)} d h
$$

By (6), the density function of $Z^{\alpha} / \phi=k$ is

$$
\frac{e^{-t g\left((\phi k)^{1 / \alpha}\right)-(\phi k)^{1 / \alpha}}}{\Gamma(\alpha+1)} d k .
$$

Since the moment problem is determined for $\alpha \leqq 2$ (cf. [1]), we have that

$$
k^{1 / \alpha}\left[\frac{1-\phi^{1 / \alpha}}{t}\right]=g\left((\phi k)^{1 / \alpha}\right)
$$

or 


$$
g(x)=\left[\frac{1-\phi^{1 / \alpha}}{t \phi^{1 / \alpha}}\right] x=c x .
$$

We now see, combining the preceding theorems, that in the case under consideration, if the Cauchy functional equation (2) is satisfied, then the function must be $g(x)=c x$, where $c$ is a constant.

The following statistical result may be proved using the preceding theorem.

Theorem 3. Let $X_{1}, X_{2}, \cdots$ be a sequence of non-negative independent random variables with the same continuous distribution function $F(x)$, and let $N_{x}$ be defined as follows:

$$
N_{x}=\left\{\begin{array}{l}
0 \text { if } X_{1}>x \\
n \text { if } X_{1}+X_{2}+\cdots+X_{n} \leqq x \\
\quad \text { and } X_{1}+X_{2}+\cdots+X_{n+1}>x .
\end{array}\right.
$$

If $N_{x}$ has a distribution function of the form

$$
\begin{aligned}
\operatorname{Pr}\left\{N_{x} \leqq n\right\}=e^{-f(x)}[1+f(x) & +\frac{1}{2 !} f^{2}(x)+\cdots \\
& \left.+\frac{1}{[(n+1) \alpha-1] !} f^{[(n+1) \alpha-1]}(x)\right],
\end{aligned}
$$

with fixed $\alpha=1$ or 2 , for every positive $x$, then $F(x)$ is of the gamma type

$$
F(x)= \begin{cases}0 & \text { for } x<0, \\ \int_{0}^{x} \frac{\omega^{\alpha-1}}{\Gamma(\alpha) \beta^{\alpha}} e^{-\omega / \beta} d \omega & \text { for } x \geqq 0 .\end{cases}
$$

Mr. Seiji Nabeya [2] has obtained a proof for the case $\alpha=1$ when $F(x)$ is not assumed to be continuous.

\section{REFERENCES}

1. J. A Shohat and J. D. Tamarkin, The problem of moments, Mathematical Surveys, no. 1, New York, American Mathematical Society, 1943.

2. Seiji Nabeya, On a relation between exponential law and Poisson's law, Annals of the Institute of Statistical Mathematics vol. 2, no. 1 (1950).

The University of Chicago 